\title{
Social Ties and Cognitive Recovery after Stroke: Does Social Integration Promote Cognitive Resilience?
}

\author{
M. Maria Glymour ${ }^{a}$ b Jennifer Weuve ${ }^{b}$ Martha E. Fay ${ }^{b}$ Thomas Glass ${ }^{c}$ \\ Lisa F. Berkman ${ }^{\text {b }}$ \\ ${ }^{a}$ Columbia University Mailman School of Public Health, New York, N.Y., ${ }^{b}$ Harvard School of Public Health, \\ Boston, Mass., and 'Johns Hopkins Bloomberg School of Public Health, Baltimore, Md., USA
}

\section{Key Words \\ Social support $\cdot$ Brain infarction $\cdot$ Cognitive reserve $\cdot$ \\ Cognitive disorders $\cdot$ Neuropsychological tests $\cdot$ Causality}

\begin{abstract}
Background/Aims: Little is known about the possible effects of social resources on stroke survivors' level and change in cognitive outcomes. Understanding this association may help us identify strategies to improve stroke recovery and help elucidate the etiology of dementia. Methods: We examined the relationship of social ties and social support to cognitive function and cognitive change 6 months after stroke. Participants in the Families in Recovery from Stroke Trial (FIRST) ( $n=272$ ) were interviewed approximately 17 days (baseline) and 6 months (follow-up) after stroke. Cognition was assessed with the Mini Mental State Examination (MMSE) and a summary battery of 7 neuropsychological tests. Median-based regression was used to model cognitive outcomes by level of baseline intimate, personal and organizational social ties and received emotional and instrumental support. Results: Baseline social ties and emotional support independently predicted 6-month Cognitive Summary Scores. Emotional support also predicted greater improvements in Cognitive Summary Scores from baseline to the 6-month follow-up. No other social exposures predicted
\end{abstract}

\begin{tabular}{ll}
\hline KARGER & ๑ 2008 S. Karger AG, Basel \\
$\begin{array}{l}\text { Fax }+41613061234 \\
\text { E-Mail karger@karger.ch } \\
\text { www.karger.com }\end{array}$ & $\begin{array}{l}\text { Accessible online at: } \\
\text { www.karger.com/ned }\end{array}$
\end{tabular}

improvements in the MMSE or the Cognitive Summary. Conclusions: Our results suggest that emotional support may promote cognitive resilience while social ties provide cognitive reserve that protects against impaired cognition after stroke. Social ties did not predict cognitive recovery however, so reverse causation cannot be ruled out.

Copyright $\odot 2008$ S. Karger AG, Basel

\section{Introduction}

Stroke is a leading cause of cognitive impairment in the elderly, but many stroke survivors retain normal cognitive function [1]. Identifying determinants of stroke survivors' cognitive outcomes may enrich our understanding of both stroke recovery and the etiology of dementia. Given the role of vascular factors in cognitive aging [2], characteristics that promote resilience or vulnerability of cognitive function after stroke may also influence cognitive aging and dementia in populations without clinical stroke.

The relationship between aspects of social connections and cognitive function and recovery after stroke is a promising but not well-understood area. Although evidence suggests that social contacts and social support improve physical recovery after stroke (reviewed in Berk-
M. Maria Glymour, ScD

Department of Epidemiology

Mailman School of Public Health at Columbia University

722 West 168th Street, Room 1603, New York, NY 10032 (USA)

Tel. +1 212342 0166, Fax +1 212342 5169, E-Mail mglymour@hsph.harvard.edu 
man and Glass [3]), this work has only rarely been extended to cognitive outcomes [4]. Social integration may promote cognitive recovery through several mechanisms. The cognitive demands of social interactions, such as receptive and expressive communication, recall of shared experiences and problem solving, may have direct benefits for neurologic function. Members of close social networks may also encourage patients to engage in healthpreserving behaviors (e.g. medication adherence). Finally, social interactions and social support may offset harm to physical and cognitive function arising from highly stressful events.

Prior studies of cognitive aging link social connections and support to maintenance of cognitive function and prevention of dementia in elderly populations (reviewed in Fratiglioni et al. [5]), suggesting that social engagement may facilitate cognitive resilience. The evidence linking social ties and cognitive decline in the elderly is suggestive but not conclusive. An important critique of this research has been difficulty in establishing a temporal order, i.e. cognitive declines may lead to disengagement from social resources [6]. It has been very difficult to identify the time of onset of cognitive changes and thus to establish that social ties measures precede the cognitive changes. Examining the relationship between social resources and cognitive recovery among stroke survivors helps circumvent this problem: the process of cognitive recovery begins only after the stroke, thus measures of social connections at the time of the stroke or immediately thereafter are unlikely to be influenced by the extent of recovery.

We hypothesize that stroke survivors with more extensive social ties and greater emotional and instrumental social support immediately after stroke will experience greater improvements in cognitive function over 6 months of follow-up and achieve a higher level of cognitive functioning 6 months after stroke. We examine these hypotheses in the Families in Recovery from Stroke Trial (FIRST), a randomized trial of 291 poststroke patients, analyzed here as a prospective observational cohort of stroke survivors.

Social network research contrasts the benefits of different types of ties [3]. We consider 3 domains of social integration: intimate ties, other personal relationships, and social engagement (voluntary affiliations or activities). In addition to examining structural aspects of social networks (number and types of ties), which are unlikely to change immediately after stroke onset, we also measured received emotional and instrumental support. Structural measures of ties offer the best opportunity to determine whether social integration - independent of stroke characteristics - alters the course of recovery. Social support may more directly measure the pathways through which social connections affect health.

\section{Methods}

\section{Sample}

The FIRST was a randomized trial of a psychosocial intervention targeting functional recovery after stroke [7]. Patients aged 45 years or older admitted to 8 Boston area hospitals and rehabilitation facilities were eligible if they met National Institute of Neurologic Diseases and Stroke criteria for ischemic or nontraumatic hemorrhagic stroke. Exclusion criteria were: limited comprehension and expressive capability; extreme social isolation; residence in a nursing home prior to stroke or at discharge; prestroke cognitive impairment; residence outside of metropolitan Boston, or impairment from the stroke either too mild or too severe, i.e. NIH Stroke Severity (NIHSS) index $<3$ or $>8$, developed in Adams et al. [8]. All randomized patients $(n=291)$ provided written informed consent. Baseline interviews were conducted shortly after index stroke and before randomization. Subsequent interviews were scheduled for 3 and 6 months later (with 2-week windows). Although the FIRST was a randomized trial, the psychosocial intervention had little effect on social ties [9] and cannot be used to test our hypotheses. Our analyses disregard randomization and treat the data as a prospective observational cohort.

\section{Measures}

Social ties and received social support were assessed at the initial interview, an average of 17-20 days after stroke. Social support was measured using a modified version of Barrera's Inventory of Socially Supportive Behaviors [10], with 7 emotional support items (e.g. 'In the last month, how often did someone tell you that they care about you?') and 5 instrumental support items (e.g. 'In the last month, how often did someone help you with household chores such as cooking, doing laundry or cleaning up?'). Responses on a 5-point frequency scale (from 'not at all' to 'every day') were summed. For ease of interpretation of the parameter coefficients, the support variables were transformed to $\mathrm{z}$-scores by subtracting the mean and dividing by the standard deviation. We did not combine the instrumental and emotional support measures because of evidence that these dimensions of social support have qualitatively different effects on stroke recovery [11]. We did not use the informational support domain of Barrera's inventory because this was strongly associated with measured stroke severity on the NIHSS. Emotional and instrumental supports were independent of baseline NIHSS after age adjustment. Individuals who did not answer the social support questions $(\mathrm{n}=43)$ were retained in the model using the missing indicator method.

To assess social ties, we created 3-point measures of intimate ties, other personal ties, organizational ties and a summary social ties measure. The intimate ties index assigned 1 point each for spouses/live-in partners and confidantes seen at least weekly. The personal ties index assigned 1 point each if patients reported ties with 1 or more children, 2 or more friends and 2 or more relatives; 
the 2 most isolated categories were combined. Participants who refused questions on a type of tie were considered not to have that tie. Organizational ties were assessed with questions about unpaid volunteer or community work, paid work, religious attendance and attendance at civic organizations (nonchurch). The organizational ties scale assigned 1 point each for up to 2 types of ties. Questions were phrased 'In the last month have you...' with yes/no responses. Thus, the referenced time periods spanned stroke onset date. We created a summary Social Ties Index by counting the domains (intimate, personal, organizational) in which patients scored above zero. The 2 lowest categories of the index were combined, giving a range of $0-2$ on the summary index and each subcomponent index. This resulted in somewhat unequal numbers of participants in each category, with the fewest individuals in the least connected group. Because of the limited granularity of the social network items, alternative categorizations that achieve more even sample sizes in each group combine individuals with substantively distinct levels of social integration.

Cognitive function was assessed at baseline and the 6-month follow-up using the Mini Mental State Examination (MMSE) and a battery of 7 neuropsychological examinations. For each cognitive test, we considered 2 outcomes: cognitive function at 6-month interview, and change in cognitive function between baseline and 6 months. The MMSE has a possible range of 0-30 [12]. Up to 5 missing items were considered errors; respondents who refused or skipped $>5$ items were considered missing the MMSE.

The cognitive battery included the Wechsler Digit Span Forward (revised to assess attention) [13], Boston Diagnostic Aphasia Examination Repetition and Comprehension (combined, possible range 0-12) [14], Immediate Recall of a 10-word list, Delayed Word Recall (11-min average delay), the 1-min Animal Naming test and Trailmaking tests A and B. We present results for each test separately and for a combined summary cognitive measure. Trailmaking tests [15] were administered following the Reitan assessment method [16], and lower times indicate better performance. The Trails B test was usually interrupted after $300 \mathrm{~s}$. Respondents who exceeded $300 \mathrm{~s}$, quit without completing the task or completed Trails A, but not Trails B, were assigned the worst possible score of 301. In addition to considering each cognitive domain separately, we constructed a cognitive summary measure to provide a broad assessment of cognitive performance in multiple domains. Using an approach similar to that found in prior research [17], we constructed the cognitive summary measure by averaging z-scores for each instrument (see Lezak [16] for background on the neuropsychological measures). The Cognitive Summary Score was correlated 0.70 with the MMSE score at the 6-month assessment. All of the cognitive outcomes were modeled as continuous variables.

Analyses

Several of the cognitive tests have artificial ceilings and floors, a measurement problem that can seriously bias ordinary least squares regression coefficient estimates of the effect of social ties (or any other exposure) on cognitive function or change $[18,19]$. To avoid this bias, we used censored least absolute deviations (CLAD) regression models. CLAD estimate differences in the $m e-$ dian (or other quantile) outcome across levels of the exposure, in contrast to ordinary least squares which estimate differences in means [20]. Analyses used a modified version of the CLAD pro- gram, implemented in Stata 8 [21] (code available from authors). CLAD coefficients are interpreted similarly to conventional regression coefficients: the coefficient is the predicted difference in median outcome value per unit increase in the exposure variable. CLAD estimates do not converge if the median value of the outcome is equal to the ceiling, which was the case for the aphasia measure. For this outcome, we specified estimation for the effect of the independent variables on the 25th percentile. Confidence intervals (CIs) were bootstrapped by resampling individuals (500 resamples) instead of observations, in order to handle correlations between repeated measures on the same individual. We present bias-corrected 95\% CIs. If the 95\% CI does not include the null value of zero, we interpret this as evidence of statistical significance; borderline significance is noted if less than $8 \%$ of the width of the $95 \% \mathrm{CI}$ extends beyond the null value (this value approximately corresponds to a $90 \% \mathrm{CI}$ or a $\mathrm{p}<0.10$ if the estimator follows a normal distribution).

We first present estimates of the association between each social ties measure and cognitive outcomes at 6 months after stroke. When there was evidence of a statistically significant association, we also tested for modification by age, using an interaction term between the social ties measure and age dichotomized at the sample median (71 years). To identify predictors of change, we next estimated models using data that included both the baseline and follow-up outcome value. All models included the social ties variable, an indicator for assessment wave (baseline vs. follow-up) and the interaction term (product) of these two variables. We interpreted the coefficient of the interaction term as the effect of the social variable on the amount of recovery between baseline and follow-up, as in simple growth curve models. This coefficient could also be interpreted as the effect of the social variable on the rate of recovery, because the period of time between baseline and 6-month follow-up was very similar for all patients.

To ameliorate bias potentially arising from missing 6-month cognitive scores, analyses were inverse probability weighted [22]. We calculated inverse probability weights using the covariates included in each regression model, plus baseline Augmented Barthel Index (measuring activities of daily living independence), baseline value of the cognitive outcome, baseline MMSE (whether or not MMSE was the outcome) and baseline Cognitive Summary Score.

All models were adjusted for age and education $(<12,12$ or $>12$ years). Age was modeled using linear splines with a knot at the age of 70, which allowed the slope of change associated with an extra year of age to be estimated separately for ages under 70 and ages 70 and above. Spline models are often preferable to alternative approaches to controlling for continuous confounders, such as converting to categorical indicators or using arbitrary polynomials [23]. Age and education were forced into all models because of their strong relations with cognitive outcomes. Regression coefficients are also presented after adjustment for additional covariates including: race (white/all other), sex, self-reported household income (USD $<15,000$ per year, USD $\geq 15,000$ or missing), a comorbidity index (based on medical record review of the number of the following conditions present: myocardial infarction, vascular disease, pulmonary disease, endocrine disease, renal or liver disease, gastrointestinal disease, cancer, rheumatologic disease, psychiatric condition or other serious debilitating chronic condition), diabetes, lesion side (left/all other), cortical stroke and baseline NIHSS. 
Table 1. Characteristics of FIRST participants, by summary Social Ties Index

\begin{tabular}{|c|c|c|c|}
\hline \multirow[t]{2}{*}{ Characteristic } & \multicolumn{3}{|c|}{ Social Ties Index } \\
\hline & score 0 & score 1 & score 2 \\
\hline Full sample & $60(100)$ & $119(100)$ & $93(100)$ \\
\hline Age at stroke, years & $72.2 \pm 11.3$ & $69.9 \pm 10.1$ & $66.3 \pm 11.3$ \\
\hline White race/ethnicity & $50(83)$ & $107(90)$ & $77(83)$ \\
\hline Female* $^{*}$ & $35(58)$ & $58(49)$ & $39(42)$ \\
\hline \multicolumn{4}{|l|}{ Level of education* } \\
\hline$<12$ years & $26(43)$ & $29(24)$ & $18(19)$ \\
\hline 12 years & $17(28)$ & $43(36)$ & $29(31)$ \\
\hline$\geq 13$ years & $17(28)$ & $47(39)$ & $46(49)$ \\
\hline Household income USD 15,000 or more & $18(30)$ & $41(34)$ & $37(40)$ \\
\hline Diabetes* & $24(40)$ & $38(32)$ & $19(20)$ \\
\hline Left-sided stroke & $21(35)$ & $36(30)$ & $26(28)$ \\
\hline Cortical stroke & $13(22)$ & $33(28)$ & $18(19)$ \\
\hline Comorbidity score & $3.83 \pm 2.45$ & $3.64 \pm 2.19$ & $3.49 \pm 2.28$ \\
\hline Baseline NIHSS score & $6.0 \pm 3.6$ & $6.1 \pm 4.1$ & $6.2 \pm 4.0$ \\
\hline Emotional support & $-0.49 \pm 0.98$ & $0.08 \pm 1.00$ & $0.22 \pm 0.92$ \\
\hline Instrumental support & $-0.44 \pm 0.79$ & $0.03 \pm 1.03$ & $0.22 \pm 0.94$ \\
\hline Stroke to baseline assessment, days & $17.3 \pm 7.9$ & $17.9 \pm 7.6$ & $16.4 \pm 7.1$ \\
\hline Stroke to 6-month assessment, days & $204.9 \pm 8.2$ & $205.5 \pm 7.5$ & $203.4 \pm 7.7$ \\
\hline
\end{tabular}

Results are means \pm standard deviation or numbers with percentages in parentheses. The Social Ties Index is a combined measure of intimate, personal or organizational ties, based on the number of domains in which the participant had some ties, with the bottom two categories combined. ${ }^{*} \mathrm{p}<0.05$ : significantly associated with the social ties variable.

Respondents missing data on social ties $(n=10)$ or comorbidity index $(n=9)$ were excluded from all analyses. Data from all of the remaining 272 subjects were used to calculate inverse probability weights to account for missing values of the 6-month cognitive test scores; baseline characteristics of all these people are presented in table 1 . Other exclusions in analyses of the 6 -month outcomes were patients who died prior to the final interview $(n=12)$, were too sick to be interviewed $(n=6)$, refused to continue in the study $(n=7)$ or refused follow-up cognitive testing. Because of the small sample size, we included all respondents with data on that outcome for each assessment, even if the respondent did not complete the other cognitive outcome tests. The analyses of cognitive function at 6 months after stroke used data on 242 MMSE respondents, 234 cognitive summary respondents and ranged from 212 (Trails B) to 231 (Digit Span) for the component cognitive tests. Respondents with valid outcome scores at either baseline or follow-up were included in the corresponding analyses of change.

\section{Results}

The sample was $49 \%$ female, with an average age of 70 years (range 45-93). Characteristics of sample members, stratified by score on the summary Social Ties Index, are presented in table 1. Baseline cognitive assessments oc- curred an average of 17 days after stroke, and 6-month follow-ups were conducted an average of 205 days after stroke. The Social Ties Index correlated with received emotional (Spearman's $\mathrm{r}=0.25, \mathrm{p}<0.01$ ) and instrumental support $(\mathrm{r}=0.24, \mathrm{p}<0.01)$. Emotional and instrumental supports were also correlated with each other $(r=0.27$, $\mathrm{p}<0.01)$. The NIHSS did not predict the Social Ties Index $(p=0.73)$, emotional support $(p=0.76)$ or instrumental support $(\mathrm{p}=0.47)$.

The FIRST was a randomized trial of a psychosocial intervention intended to improve social networks and social support for stroke survivors and ultimately benefit functional recovery from stroke. This would provide an ideal opportunity to test the hypothesis that social ties benefit cognitive recovery from stroke if the intervention had successfully changed the key aspects of social relationships. However, as reported elsewhere, the trial did not appear to change any of the social ties variables that we hypothesized might affect cognitive recovery $[9,24]$. There were no significant differences between treatment groups with respect to social ties measured at 6-month follow-up ( $p=0.38$ for intimate ties, $p=0.64$ for personal ties, $\mathrm{p}=0.13$ for organizational ties, $\mathrm{p}=0.18$ for Social 
Table 2. Distribution of cognitive outcomes among FIRST participants

\begin{tabular}{|c|c|c|c|c|c|c|c|c|}
\hline Tests & & $\mathrm{n}$ & Mean $\pm \mathrm{SD}$ & $\begin{array}{l}\text { 25th } \\
\text { percentile }\end{array}$ & Median & $\begin{array}{l}\text { 75th } \\
\text { percentile }\end{array}$ & $\begin{array}{l}\text { At measure- } \\
\text { ment floor }\end{array}$ & $\begin{array}{l}\text { At measure- } \\
\text { ment ceiling }\end{array}$ \\
\hline \multirow[t]{2}{*}{ MMSE } & Baseline & 259 & $24.97 \pm 3.55$ & 22.0 & 25.0 & 27.0 & $0(0)$ & $12(5)$ \\
\hline & 6 months & 242 & $26.05 \pm 3.56$ & 25.0 & 27.0 & 28.0 & $0(0)$ & $28(12)$ \\
\hline Cognitive Summary & Baseline & 249 & $-0.03 \pm 0.68$ & -0.5 & 0.0 & 0.3 & n.a. & n.a. \\
\hline Score & 6 months & 234 & $-0.02 \pm 0.71$ & -0.5 & -0.1 & 0.4 & n.a. & n.a. \\
\hline \multirow[t]{2}{*}{ Digit Span Forward } & Baseline & 249 & $8.30 \pm 2.59$ & 6.0 & 8.0 & 10.0 & $2(1)$ & $20(8)$ \\
\hline & 6 months & 231 & $8.65 \pm 2.36$ & 7.0 & 9.0 & 11.0 & $1(0)$ & $18(8)$ \\
\hline \multirow[t]{2}{*}{ BDAE } & Baseline & 246 & $11.09 \pm 1.32$ & 10.0 & 11.0 & 12.0 & $0(0)$ & $132(54)$ \\
\hline & 6 months & 214 & $11.15 \pm 1.26$ & 11.0 & 12.0 & 12.0 & $0(0)$ & $115(54)$ \\
\hline \multirow[t]{2}{*}{ Immediate Recall } & Baseline & 241 & $5.05 \pm 1.61$ & 3.7 & 5.0 & 5.7 & $2(1)$ & $0(0)$ \\
\hline & 6 months & 220 & $5.48 \pm 1.69$ & 4.3 & 5.7 & 6.7 & $0(0)$ & $0(0)$ \\
\hline \multirow[t]{2}{*}{ Delayed Recall } & Baseline & 241 & $3.70 \pm 2.58$ & 1.0 & 3.0 & 5.0 & $42(17)$ & $2(1)$ \\
\hline & 6 months & 229 & $4.43 \pm 2.45$ & 3.0 & 5.0 & 6.0 & $20(9)$ & $1(0)$ \\
\hline \multirow[t]{2}{*}{ Animal Naming } & Baseline & 240 & $12.46 \pm 5.61$ & 8.0 & 11.0 & 15.0 & $2(1)$ & $2(1)$ \\
\hline & 6 months & 228 & $14.00 \pm 5.56$ & 10.0 & 50.0 & 75.0 & $1(0)$ & $7(3)$ \\
\hline \multirow[t]{2}{*}{ Trailmaking A } & Baseline & 219 & $113.43 \pm 81.70$ & 55.0 & 74.5 & 110.0 & n.a. & $25(11)$ \\
\hline & 6 months & 215 & $88.47 \pm 58.21$ & 51.0 & 70.0 & 107.0 & n.a. & $8(4)$ \\
\hline \multirow[t]{2}{*}{ Trailmaking B } & Baseline & 211 & $232.63 \pm 84.21$ & 132.3 & 252.5 & 301.0 & n.a. & $104(49)$ \\
\hline & 6 months & 212 & $209.85 \pm 91.06$ & 120.3 & 224.0 & 301.0 & n.a. & $88(42)$ \\
\hline
\end{tabular}

Figures in parentheses are percentages. $\mathrm{SD}=$ Standard deviation; . $\mathrm{a} .=$ the floor or ceiling is undefined for this outcome; $\mathrm{BDAE}=$ Boston Diagnostic Aphasia Examination. In the Trailmaking Tests, lower scores indicate better performance; individuals at the measurement ceiling have the worst possible score.

Ties Index, $p=0.92$ for emotional support and $p=0.45$ for instrumental support). The remainder of this paper treats the data set as an observational study of a cohort of stroke survivors and uses social ties and social support measures at the first poststroke assessment (baseline) to predict 6-month cognitive function and the magnitude of change in cognitive function between baseline and 6 months.

A high proportion of respondents received the maximum possible MMSE score at baseline and the 6-month follow-up (table 2). There were similar measurement ceilings for the Digit Span, Boston Diagnostic Aphasia Examination and Trailmaking tests. A measurement floor was evident for the Delayed Recall test. The proportion of respondents scoring the maximum possible MMSE differed across Social Ties Index, indicating that the ceiling could bias conventional ordinary least squares regression coefficients, and the CLAD regression coefficients are more appropriate. For example, $21 \%$ of respondents with the highest social ties score reported a 6-month
MMSE of 30, versus only $5 \%$ of respondents in the lowest social ties category (results not shown).

\section{Social Integration and 6-Month Cognitive Outcomes}

Although individuals with higher baseline Social Ties Index scores and more emotional support had higher median 6-month MMSE scores, both relations are of borderline statistical significance in age- and education-adjusted models (table 3). Neither relationship remained statistically significant after full covariate adjustment. No other social ties variables were significantly associated with 6-month MMSE.

The Social Ties Index was a significant predictor of the 6-month Cognitive Summary Score, even after full covariate adjustment ( $\beta=0.21,95 \%$ CI: $0.06,0.36$ ). This relationship was not clearly attributable to intimate, personal or organizational ties; none of these component social ties measures was significantly related to the Cognitive Summary Score. The Social Ties Index was statistically significantly associated with only 2 component 


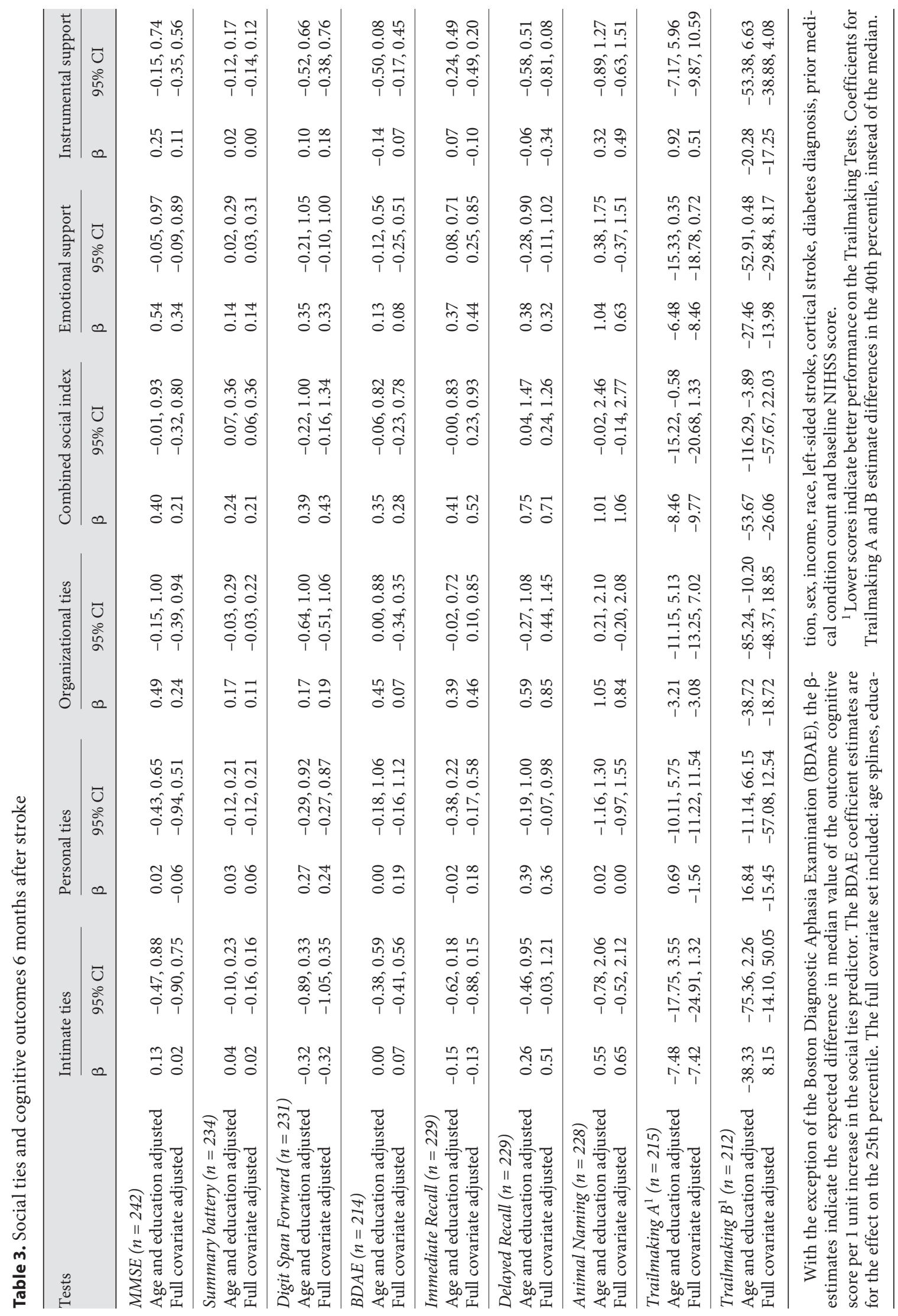




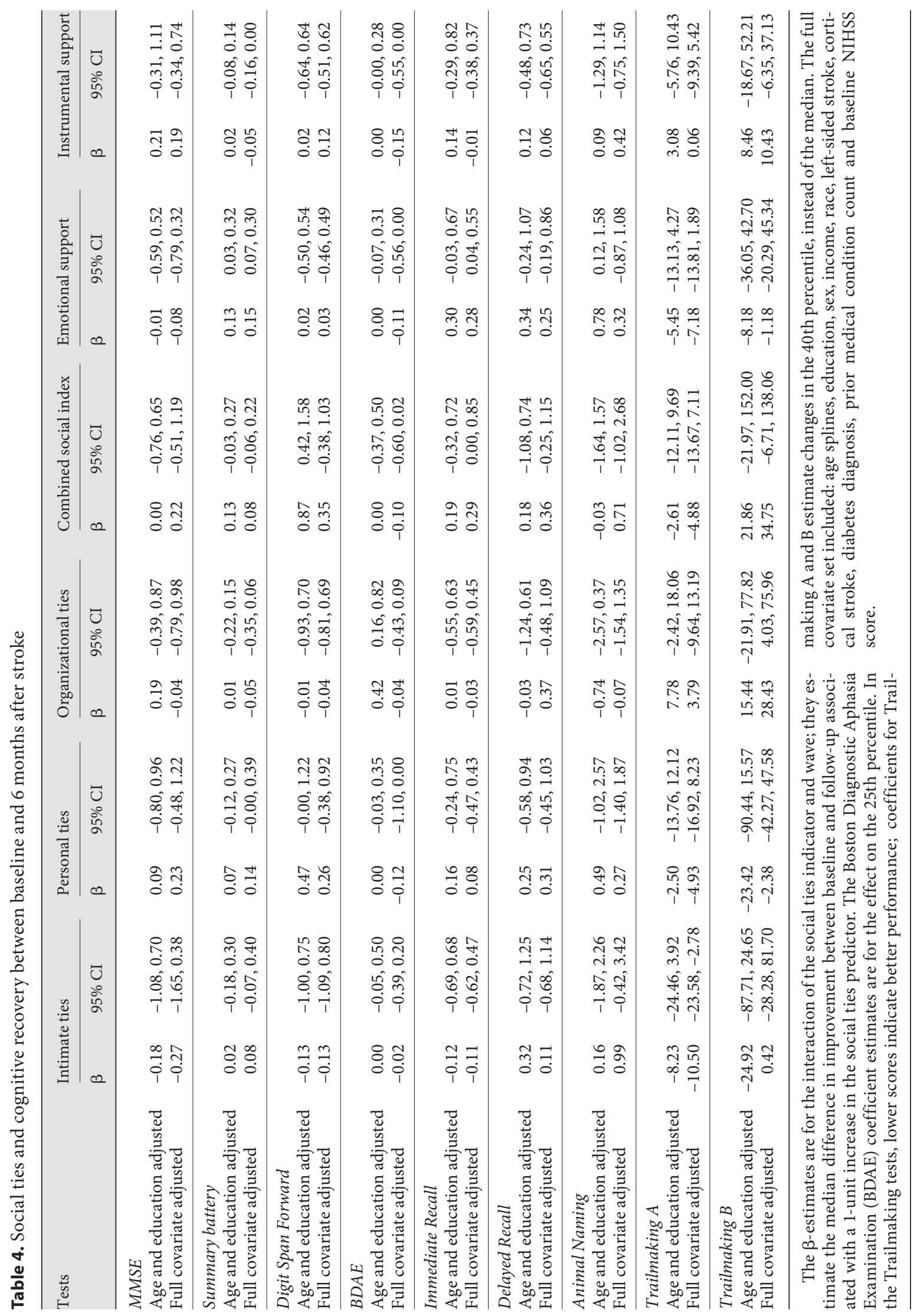


measures of the 6-month Cognitive Summary Score: Immediate and Delayed Word Recall. The associations between the Social Ties Index and Animal Naming and Trails A were both of borderline significance. There was no evidence that the associations between Social Ties and the Cognitive Summary, Immediate or Delayed Word Recall measures were modified by age.

Emotional support also predicted the Cognitive Summary Score ( $\beta=0.14 ; 95 \%$ CI: $0.03,0.31)$ after full covariate adjustment. Baseline instrumental support did not predict the 6-month Cognitive Summary Score. Because both the Social Ties Index and emotional support were significant predictors of the Cognitive Summary Score, we also tested whether emotional support might mediate the relationship between Social Ties Index and Cognitive Summary Score. In a model adjusted for all covariates, both the Social Ties Index ( $\beta=0.25$; $95 \%$ CI: $0.09,0.42)$ and emotional support ( $\beta=0.12$; 95\% CI: $0.01,0.28)$ variables retained independent relationships with the cognitive summary outcome. There was no evidence that the association between emotional support and the Cognitive Summary Score was modified by age.

\section{Social Relations and Cognitive Change between \\ Baseline and 6 Months}

None of the social ties or social support variables significantly predicted changes in MMSE between baseline and 6-month follow-up (table 4). Higher levels of emotional support predicted greater levels of recovery (change) on the Cognitive Summary Score in models with full covariate adjustment $(\beta=0.15 ; 95 \%$ CI: 0.07 , 0.30 ). This relationship did not differ significantly for individuals older or younger than 71 ( $\beta$ for interaction $=$ $-0.01 ; 95 \%$ CI: $-0.19,0.27)$. Figure 1 displays the predicted median change in Cognitive Summary Score by level of emotional support. Emotional support was significantly related to recovery in the Immediate Recall score $(\beta=0.28$; 95\% CI: $0.04,0.55)$ but not with any other component cognitive test. No other social ties variable predicted recovery on the Cognitive Summary Score.

\section{Discussion}

Our results indicate that stroke survivors who reported social ties in multiple areas and those with higher emotional support immediately after stroke have better Cognitive Summary Scores 6 months later compared to socially isolated individuals or those with less emotional support. Contrary to our hypotheses, baseline social ties

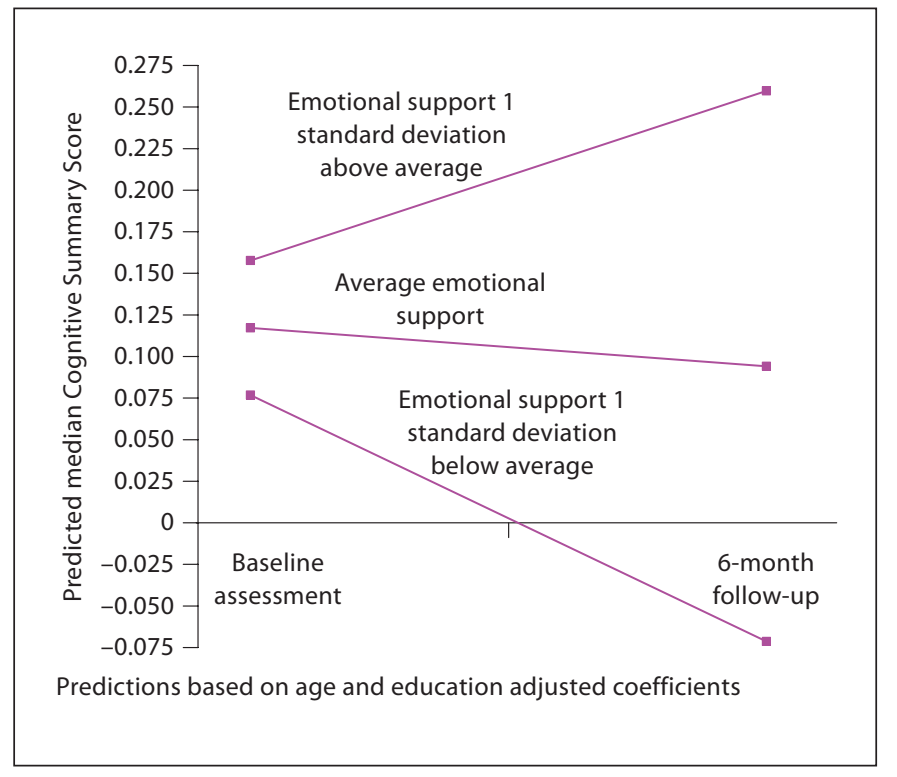

Fig. 1. Predicted trajectory of the Cognitive Summary Score, by level of emotional support.

did not predict greater recovery (improvements) during the follow-up period; in other words, the advantage evident at the 6-month follow-up was similar to the advantage that prevailed at the baseline poststroke assessment. Higher levels of emotional support at baseline predicted better cognitive recovery during the follow-up period such that a 1-standard-deviation increase in emotional support was associated with a 0.14-standard-deviationlarger improvement in the Cognitive Summary Score. These results should be interpreted cautiously in light of the study's limitations.

\section{Study Strengths and Limitations}

FIRST participants may not be representative of the population of stroke survivors. The trial excluded patients with either extreme social isolation or cognitive impairment. Comparisons with the distributions of cognitive scores of stroke survivors in the Health and Retirement Study/Aging, Demographics and Memory substudy suggest that FIRST participants are generally more highly functioning than community samples (results available from authors). If the effects of social integration and health are strongest at extreme levels of isolation, our results may underestimate relationships between social ties and poststroke cognitive outcomes. Selection based on stroke survival, differential dropout or test refusal may also bias findings, although there was not a strong asso- 
Fig. 2. Alternative causal structures consistent with an association between social ties and poststroke cognitive function.

(a) Cognitive resilience: poststroke social ties or support promote cognitive recovery

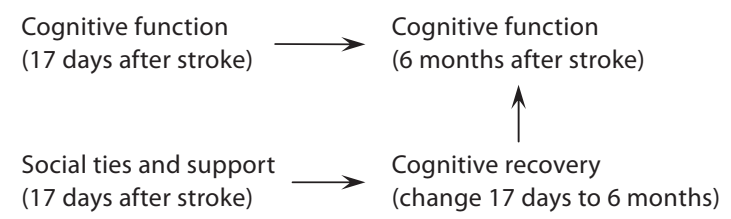

(b) Confounding by stroke severity: stroke characteristics affect both social ties and cognitive function after stroke

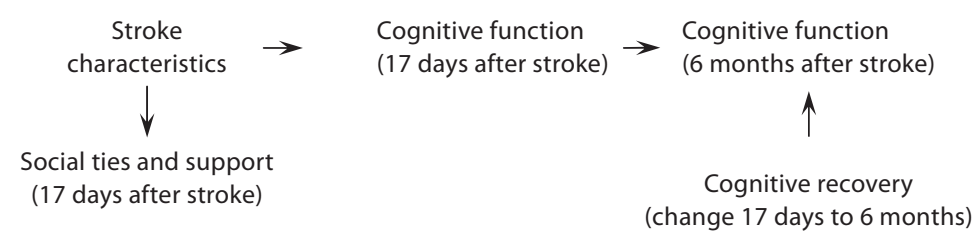

(c) Reverse causation: cognitive function prior to stroke affects social ties and cognitive function before and after stroke

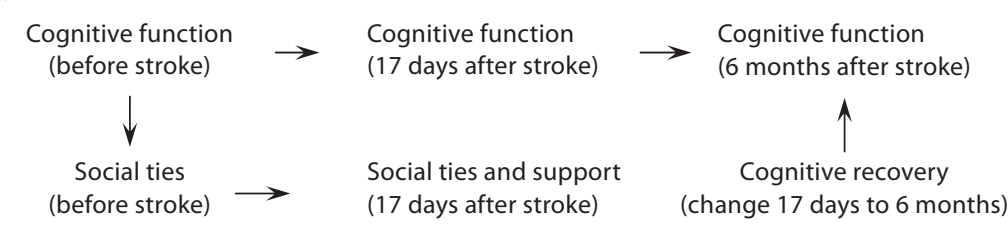

(d) Cognitive reserve: social ties prior to stroke affect cognitive function before stroke

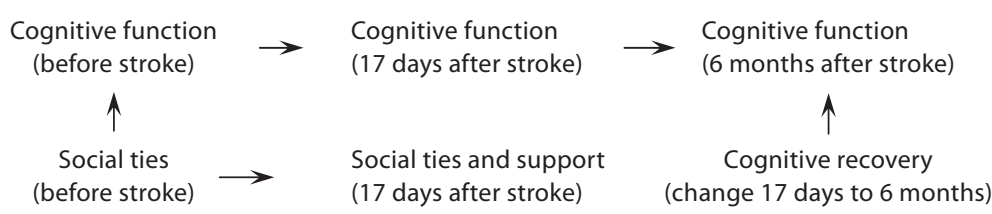

ciation between social ties and loss to follow-up. Enrollment of stroke survivors made it difficult to conduct careful cognitive assessments promptly after stroke, and recovery during the period between stroke and first cognitive assessment (averaging 17 days) could obscure effects of social ties on cognitive recovery. The relatively small sample of 272 stroke survivors is an additional limitation, reflected in wide CIs for some parameter estimates. The small sample also precludes correction for multiple-hypothesis testing, suggesting that caution is appropriate in interpreting the statistical tests of significance. However, samples of stroke survivors are typically small and replication of results in independent samples is usually necessary for confirmation.
This study also has several important strengths. We used social ties measures tapping multiple domains and assessed at baseline. Using social ties measures that temporally precede the cognitive assessments helps rule out the possibility of reverse causation. We present results on an unusually comprehensive battery of cognitive assessments. Measurement problems such as practice effects and ceilings often complicate research on cognitive outcomes [19]. Our analyses circumvented the measurement ceiling problem by focusing on changes in median performance instead of mean performance. Because the bias from measurement ceilings introduced in analyses of change is of unknown direction, this is a substantial advantage. 
Association of Social Integration and 6-Month

\section{Cognitive Function}

Compared to measures of social ties in any single domain, the summary Social Ties Index, capturing isolation in any of these 3 areas, was the most robust predictor of cognitive function 6 months after stroke. This index was similar to that used by Bassuk et al. [25] to predict cognitive function and cognitive change in a population sample of elderly individuals followed for 12 years. The relative strength of the summary Social Ties Index compared to any of the individual domains (intimate, personal or organizational) suggests a discontinuous relationship, with the absence of ties being the most important factor influencing cognitive function. We also found a robust association between emotional support and 6-month Cognitive Summary Score. Emotional support did not seem to mediate the association between Social Ties Index and Cognitive Summary Score, rather both measures were independent predictors of the outcome. This is interesting in light of prior research on social integration and cognitive aging. Social network or contact measures have more frequently been found to predict cognitive outcomes $[5,25,26]$, but some studies find emotional support to be important [27]. Our results suggest that social integration and emotional support may relate to independent processes that link to cognitive outcomes. For example, social contacts may operate through cognitive stimulation, similar to environmental enrichment in animal models $[28,29]$, while emotional support operates through stress or behavioral pathways [3].

There was no evidence that receipt of instrumental support predicted better 6-month cognitive function in any domain. Prior research on instrumental support and physical independence suggests that too much instrumental support can be harmful [11]. Reverse causation may also influence this association, if individuals with cognitive limitations require extra instrumental support.

\section{Association between Social Integration and \\ Cognitive Recovery}

Although the relationship between social ties and poststroke cognitive function suggests that social interactions promote cognitive resilience, there are other explanations for our findings as well. A stronger test of the hypothesis is provided by our assessment of the relation between social ties and cognitive change after stroke (instead of level of function at 6 months). However, our results for this outcome were mixed.

Social Ties and Cognition after Stroke
We consider 4 causal structures that could generate an association between social ties and cognitive function 6 months after stroke (fig. 2a-d). In the cognitive resilience model (fig. 2a), social interactions promote cognitive plasticity and thus recovery after stroke, producing the 6-month associations we witnessed. Our results for emotional support are most consistent with this hypothesis. Alternatively, stroke severity may affect both poststroke social connections and cognitive function (fig. 2b). This structure cannot be ruled out with certainty for social support measures, but there was no evidence that measures of stroke severity, such as the NIHSS, were correlated with emotional support. Confounding by stroke severity seems even less plausible for the social ties measures, because we selected measures likely to tap relationships as they existed before the stroke: existence of core network members [30] and engagement in organizational activities.

The association between social ties and cognitive function may exist before stroke, either because prestroke cognitive function affects social ties (i.e. reverse causation, as in fig. 2c) or because social ties prior to stroke influence prestroke cognitive function (fig. 2d). Using Stern's [31] framework for types of cognitive resilience, the model in figure $2 \mathrm{~d}$ suggests that social ties predict brain or cognitive reserve, but do not affect cognitive compensation or plasticity after stroke.

Our results for social ties are consistent with the causal structures in either figure $2 \mathrm{c}$ or d, and prior research also suggests that either structure is plausible. For example, although Bassuk et al. [25] and others found that social integration and related measures predict cognitive change, others have found the reverse causal direction to operate. Cognitive declines sometimes lead to social disengagement $[6,32]$.

\section{Conclusion}

In summary, our results suggest that emotional support may promote cognitive resilience while social ties provide cognitive reserve that protects against impaired cognition after stroke. Because social ties did not predict cognitive change, other explanations, such as reverse causation, are also possible. Stroke recovery provides an interesting window into determinants of cognitive plasticity. In the context of stroke recovery, some of the difficulties of drawing causal inferences can be avoided, because the timing of the onset of cognitive change is known. Although the results of this study should be in-

Neuroepidemiology 2008;31:10-20 
terpreted cautiously, it overcomes many of the difficulties in prior research on cognitive plasticity and points the way to future research that may illuminate the relationship between social integration and cognitive change. Specifically, the limitations of the current study could be addressed using data with both pre- and poststroke interviews, assessments immediately following stroke onset, characterization of the measurement properties of the cognitive outcomes, and large population samples of stroke survivors.

\section{Acknowledgements}

James Robins provided invaluable assistance with the analytic techniques used in this analysis. Ichiro Kawachi and James Robins also gave helpful advice on presentation and interpretation of the findings. The authors also gratefully acknowledge funding from the National Institute of Neurological Diseases and Stroke with supplemental funding by the National Institute on Aging (No. R01 NS/AG32324-01A1). Additional support for the analyses came from the National Institute on Aging (No. R01 NS32324-07), an NIH training grant (No. AG00158), and from a Robert Wood Johnson Health \& Society Program seed grant. Maria Glymour is a Robert Wood Johnson Foundation Health \& Society Scholar at Columbia University.

\section{References}

1 Kalaria RN, Ballard C: Stroke and cognition. Curr Atheroscler Rep 2001;3:334-339.

2 Hachinski V, Munoz D: Vascular factors in cognitive impairment - where are we now? Ann NY Acad Sci 2000;903:1-5.

3 Berkman LF, Glass T: Social integration, social networks, social support, and health; in Berkman LF, Kawachi I (eds): Social Epidemiology. New York, Oxford University Press, 2000, pp 137-173.

4 Stephens M, Kinney J, Norris V, Ritchie S: Social networks as assets and liabilities in recovery from stroke by geriatric patients. Psychol Aging 1987;2:125-129.

5 Fratiglioni L, Paillard-Borg S, Winblad B: An active and socially integrated lifestyle in late life might protect against dementia. Lancet Neurol 2004;3:343-353.

-6 Hultsch DF, Hertzog C, Small BJ, Dixon RA Use it or lose it: engaged lifestyle as a buffer of cognitive decline in aging? Psychol Aging 1999;14:245-263

-7 Glass TA, Dym B, Greenberg S, Rintell D, Roesch C, Berkman LF: Psychosocial intervention in stroke: Families in Recovery from Stroke Trial (FIRST). Am J Orthopsychiatry 2000;70:169-181.

-8 Adams HP Jr, Woolson RF, Clarke WR, Davis $\mathrm{PH}$, Bendixen $\mathrm{BH}$, Love $\mathrm{BB}$, Wasek $\mathrm{PA}$, Grimsman KJ: Design of the Trial of Org 10172 in Acute Stroke Treatment (TOAST). Control Clin Trials 1997;18:358-377.

-9 Glass TA, Berkman LF, Hiltunen EF, Furie K, Glymour MM, Fay ME, Ware J: The Families in Recovery from Stroke Trial (FIRST): primary study results. Psychosom Med 2004; 66:889-897.

10 Barrera M: A method for the assessment of social support networks in community survey research. Connections 1980;3:8-13.
1 Glass TA, Maddox GL: The quality and quantity of social support: stroke recovery as psycho-social transition. Soc Sci Med 1992; 34:1249-1261

12 Folstein MF, Folstein SE, McHugh PR: 'MiniMental State': a practical method for grading the cognitive state of patients for the clinician. J Psychiatr Res 1975;12:189-198.

13 Wechsler D: The Wechsler Adult Intelligence Scale - Revised. San Antonio, Psychological Corporation, 1981.

14 Naeser MA, Hayward RW: Lesion localization in aphasia with cranial computed tomography and the Boston Diagnostic Aphasia Exam. Neurology 1978;28:545-551.

15 Halstead W: Brain and Intelligence: A Quantitative Study of the Frontal Lobes. Chicago, University of Chicago Press, 1947.

16 Lezak MD: Neuropsychological Assessment, ed 3. New York, Oxford University Press, 1995.

17 Wilson RS, Bienias JL, Evans DA, Bennett DA: Religious orders study: overview and change in cognitive and motor speed. Aging Neuropsychol Cogn 2004;11:280-303.

18 Kennedy P: A Guide to Econometrics, ed 4. Cambridge, MIT Press, 1998.

19 Morris MC, Evans DA, Hebert LE, Bienias JL: Methodological issues in the study of cognitive decline. Am J Epidemiol 1999;149: 789-793.

20 Powell JL: Least absolute deviations estimation for the censored regression model. Econometrics 1984;25:303-325.

21 Jolliffe D, Krushelnytskyy B, Semykina A: Censored least absolute deviations estimator: CLAD. Stata Tech Bull 2000;58:13-16.

22 Hernán MA, Brumback B, Robins JM: Marginal structural models to estimate the causal effect of zidovudine on the survival of HIV-positive men. Epidemiology 2000;11: 561-570.
23 Brenner $\mathrm{H}$, Blettner M: Controlling for continuous confounders in epidemiologic research. Epidemiology 1997;8:429-434.

24 Ertel KA, Glymour MM, Glass TA, Berkman LF: Frailty modifies effectiveness of psychosocial intervention in recovery from stroke. Clin Rehabil 2007;21:511-522.

25 Bassuk SS, Glass TA, Berkman LF: Social disengagement and incident cognitive decline in community-dwelling elderly persons. Ann Intern Med 1999;131:165-173

26 Zunzunegui MV, Alvarado BE, Del Ser T, Otero A: Social networks, social integration, and social engagement determine cognitive decline in community-dwelling Spanish older adults. J Gerontol B Psychol Sci Soc Sci 2003;58:S93-S100.

27 Seeman TE, Lusignolo TM, Albert M, Berkman L: Social relationships, social support, and patterns of cognitive aging in healthy, high-functioning older adults: Macarthur studies of successful aging. Health Psychol 2001;20:243-255.

28 van Praag H, Kempermann G, Gage FH: Neural consequences of environmental enrichment. Nat Rev Neurosci 2000;1:191198.

29 Kramer AF, Bherer L, Colcombe SJ, Dong W, Greenough WT: Environmental influences on cognitive and brain plasticity during aging. J Gerontol A Biol Sci Med Sci 2004;59: 940-957.

30 Morgan D, Neal M, Carder P: The stability of core and peripheral networks over time. Social Networks 1997;19:9-25.

31 Stern Y: What Is Cognitive Reserve? Theory and Research Application of the Reserve Concept. Cambridge, Cambridge University Press, 2002, vol 8, pp 448-460.

32 Reichman WE, Negron A: Negative symptoms in the elderly patient with dementia. Int J Geriatr Psychiatry 2001;16:S7-S11. 